\title{
Neuropathic sensory symptoms: association with pain and psychological factors
}

This article was published in the following Dove Press journal:

Neuropsychiatric Disease and Treatment

20 May 2014

Number of times this article has been viewed

\author{
Maryam Shaygan \\ Andreas Böger ${ }^{2}$ \\ Birgit Kröner-Herwig' \\ 'Department of Clinical Psychology \\ and Psychotherapy, University \\ of Göttingen, Germany; ${ }^{2}$ Pain \\ Management Clinic at the Red Cross \\ Hospital, Kassel, Germany
}

Background: A large number of population-based studies of chronic pain have considered neuropathic sensory symptoms to be associated with a high level of pain intensity and negative affectivity. The present study examines the question of whether this association previously found in non-selected samples of chronic pain patients can also be found in chronic pain patients with underlying pathology of neuropathic sensory symptoms.

Methods: Neuropathic sensory symptoms in 306 patients with chronic pain diagnosed as typical neuropathic pain, radiculopathy, fibromyalgia, or nociceptive back pain were assessed using the Pain DETECT Questionnaire. Two separate cluster analyses were performed to identify subgroups of patients with different levels of self-reported neuropathic sensory symptoms and, furthermore, to identify subgroups of patients with distinct patterns of neuropathic sensory symptoms (adjusted for individual response bias regarding specific symptoms).

Results: ANOVA (analysis of variance) results in typical neuropathic pain, radiculopathy, and fibromyalgia showed no significant differences between the three levels of neuropathic sensory symptoms regarding pain intensity, pain chronicity, pain catastrophizing, pain acceptance, and depressive symptoms. However, in nociceptive back pain patients, significant differences were found for all variables except pain chronicity. When controlling for the response bias of patients in ratings of symptoms, none of the patterns of neuropathic sensory symptoms were associated with pain and psychological factors.

Conclusion: Neuropathic sensory symptoms are not closely associated with higher levels of pain intensity and cognitive-emotional evaluations in chronic pain patients with underlying pathology of neuropathic sensory symptoms. The findings are discussed in term of differential response bias in patients with versus without verified neuropathic sensory symptoms by clinical examination, medical tests, or underlying pathology of disease. Our results lend support to the importance of using adjusted scores, thereby eliminating the response bias, when investigating self-reported neuropathic symptoms by patients.

Keywords: self-reported neuropathic sensory symptoms, pain-related features, response bias

\section{Introduction}

Neuropathic pain is defined as "pain caused by a lesion or disease affecting the somatosensory system,"1 and is manifested by sensory signs and symptoms such as hyperalgesia and burning and prickling sensations. Boureau et $\mathrm{al}^{2}$ stated that verbal descriptors of experienced sensory symptoms reliably distinguish neuropathic pain from other types of pain. Several studies, however, have found similar self-reported sensory symptoms in otherwise diagnosed pain such as fibromyalgia (FM). ${ }^{3}$

A large number of population-based studies of chronic pain have reported a high level of pain intensity as well as anxiety and depressive symptoms in respondents who score high on neuropathic sensory symptoms assessed by self-report. ${ }^{4-7}$ These studies
Phaygan

Department of Clinical Psychology and

Goßlerstraße 14, 37073 Göttingen,

$\mathrm{Tel}+4955 \mathrm{I} 3933717$

Fax+4955I 393544

Email maryam.shaygan@psych.unigoettingen.de 
concluded that the neuropathic characteristics of pain are denoted by a high level of intensity as well as negative affectivity. However, some clinical studies do not support these findings when comparing medically diagnosed neuropathic and non-neuropathic pain. ${ }^{8-10}$ Consequently, two questions have been raised. First, do neuropathic sensory symptoms assessed by screening tools reliably distinguish neuropathic pain from non-neuropathic types of pain? The present study assessed self-reported neuropathic sensory symptoms in patients with "typical neuropathic pain" (TNP), radiculopathy (RAD), FM, and nociceptive back pain (nBP). We expected that these symptoms would distinguish TNP not from RAD and FM but from nBP. RAD is caused by compression or lesion of a dorsal root or its ganglion and considered as a syndrome with both nociceptive and neuropathic components of pain (mixed pain syndrome). ${ }^{11-13}$ Although in the majority of FM patients, no nerve lesions can be demonstrated, the presence of neuropathic sensory symptoms (eg, allodynia and hyperalgesia) in these patients can be explained in terms of pathogenic mechanisms such as impaired small fiber function and a dysfunction of endogenous systems modulating afferent activity. ${ }^{14-16}$ In a more recent study, Uceyler et al ${ }^{14}$ suggested a neuropathic nature of pain in FM syndrome. However, the classification of FM as neuropathic pain is a subject of controversy and debate among researchers. ${ }^{1,17}$

The second question was whether the association between a high score of self-reported neuropathic sensory symptoms with a high level of pain intensity and negative affectivity previously found in population-based studies can also be found in chronic pain patients with underlying pathology of neuropathic sensory symptoms such as TNP, RAD, and also FM.

Symptom reports are well known to be influenced by a general negative response tendency, revealed by a dominance of responses at the negative pole of a rating scale which is based on a person's disposition to disclose and report negative aspects of oneself, including both emotional and physical symptoms. In this context, a general negative self-appraisal seems to play an important role. Such biases may inflate correlations among symptoms. ${ }^{18}$ However, later studies have suggested a considerable variation in how strongly different symptoms are influenced by this response bias. For example, response bias has been suggested to more strongly relate with the report of symptoms without any identified pathology than with symptoms that can be verified by clinical examination or medical tests. ${ }^{19-21}$

In conclusion, it is argued that not only do the characteristics of pain determine the symptom report but also an individual tendency to select specific response categories, in this case preferring the endpoints of a response scale independent of the item content. This could have determined the positive correlation between selfreported neuropathic sensory symptoms with pain intensity and negative affectivity found in large population-based studies. This association should be examined in patients with an underlying pathology of neuropathic sensory symptoms.

In the present study, we wanted to analyze the relation between the level of neuropathic sensory symptoms and other pain-related parameters like intensity and chronicity as well as psychological factors (depression, catastrophizing, and pain acceptance) in four above mentioned diagnostic groups (ie, TNP, RAD, FM, and nBP). Cluster analysis was used to classify patients of these diagnostic groups based on self-reported intensity of neuropathic sensory symptoms (clustering 1). We hypothesized that only nBP patients who scored high on neuropathic sensory symptoms would report a high level of pain-related features and also psychological factors driven by the previously explained response bias. In all other diagnostic groups, significant associations were not expected.

To control for the response bias, a second clustering approach (clustering 2) based on adjusted scores (adj) of neuropathic sensory symptoms using individual means (Ms) was performed. This enabled us to subgroup the patients of the different diagnostic groups based on their distinct patterns of neuropathic sensory symptoms after having eliminated the individual response bias regarding the symptoms. Studies concerning the association of different patterns of neuropathic sensory symptoms with different parameters of pain and psychological factors are rare. A recent symptom-based study on chronic low back pain demonstrated no association between distinct patterns of neuropathic sensory symptoms with depression and anxiety. ${ }^{22}$ We wanted to find out whether the different diagnostic groups in our study were characterized by specific patterns of neuropathic sensory symptoms (after elimination of the general response bias). We expected that these symptom patterns should be represented differently in the diagnostic groups. However, since the general response bias was eliminated, none of the symptom patterns should have been associated with higher levels of pain-related parameters or psychological factors.

\section{Methods}

This cross-sectional study was conducted in a multidisciplinary tertiary care center, comprising experts in pain medicine, psychology, and neighboring professions. Inpatients presenting with chronic pain, diagnosed by anesthesiologists/neurologists, were asked to complete 
various questionnaires, after having signed informed consent regarding their participation in the study. Ethical approval was obtained from the Ethics Committee of the Georg-Elias-Müller Institute of Psychology.

\section{Sample selection}

From April 2012 to February 2013, 344 patients suffering from a chronic pain condition were referred to the pain treatment center. Chronic pain conditions included TNP, $\mathrm{RAD}, \mathrm{FM}$, and $\mathrm{nBP}$, assessed by the pain specialists who determined the pain diagnoses based on history, clinical bedside examinations, and whatever diagnostic methods were considered appropriate (eg, electrophysiological evaluation and imaging techniques). A total of 78 patients with one of the following neurological syndromes were included in the study: postherpetic neuralgia, which was confirmed if patients had persistent pain in an area previously affected by acute herpes zoster rash; complex regional pain syndrome type II, according to clinical criteria; ${ }^{23}$ central neuropathic pain, defined as pain caused by a demonstrable lesion in the central nervous system in an area anatomically attributable to the lesion; polyneuropathy, according to clinical criteria; ${ }^{24}$ and trigeminal neuralgia, according to International Headache Classification criteria 2003. ${ }^{25}$ Patients with chronic lower back pain were divided into two groups: with (RAD) or without (nBP) typical dermatomal pain (radiating beyond the knee) as well as clinical signs of nerve root involvement, including pain evoked by stretching of the femoral or sciatic nerve, sensory or motor deficits in the leg, and a decrease or loss of tendon reflexes. Moreover, available results of spinal imaging and further investigations such as electromyography were also taken into account. FM was diagnosed on the basis of the American College of Rheumatology criteria. ${ }^{26}$ Of the 344 patients, 306 were considered for inclusion in the study. Criteria for inclusion were: age above 18 years and having a chronic pain condition according to ICD-10 criteria (F45.41 or R52.1-2, International Statistical Classification of Diseases and Related Health Problems, 2012). ${ }^{27}$ The following exclusion criteria were applied: a pain history of less than 6 months, presence of a malignant disease, severe medical or psychiatric illness interfering with the pain assessment, another painful disorder or neurological disease that might have interfered with the pain assessment, and inability to comprehend the German language.

\section{Data assessment}

In addition to standard demographic inquiry, the questionnaires were applied.
Neuropathic sensory symptoms were assessed by the Pain DETECT Questionnaire (PDQ). ${ }^{4}$ The PDQ is a self-report questionnaire including nine items asking about the intensity and quality of pain. The questions address the presence of seven sensory symptoms rated on a $0-5$ rating scale (never to very strong): 1) burning pain, 2) paresthesias, 3) mechanical allodynia, 4) spontaneous pain attacks, 5) thermal hyperalgesia, 6) numbness, and 7) pressure hyperalgesia. The PDQ also comprises two questions regarding the course of pain and radiation pain. The scale was validated in a sample of patients with either neuropathic pain, including postherpetic neuralgia, polyneuropathy, nerve trauma, and lower back pain (where the source of pain is in lumbar vertebrae, sacrum, and/or coccyx), or nociceptive pain, including visceral pain, osteoarthritis, inflammatory arthropathies and non-neuropathic low back pain. The instrument indicated sensitivity as well as specificity of $84 \%$ when identifying patients with medically diagnosed neuropathic pain. The questionnaire demonstrated adequate internal consistency $($ Cronbach's alpha $=0.83) .{ }^{4}$

The average pain intensity was assessed by an eleven-point numeric rating scale ${ }^{28}$ ranging from 0 (no pain) to 10 (worst imaginable pain).

Pain chronicity was assessed by the Mainz Pain Staging System, ${ }^{29}$ which defines three stages of pain chronicity based on ten questions (in terms of four axes). Patients were requested to describe the occurrence of pain, pain duration, and changes in pain intensity (axis 1, temporal dimension); pain distribution (axis 2, spatial dimension); drug use and number of previous drug withdrawals (axis 3, drug-taking behavior); and change of personal physician, pain-related hospitalizations, pain-related operations, and pain-related rehabilitation (axis 4, utilization of the health care system). Patients were assisted by a physician to complete the questions. The sum of the four axes varies in the range of 4-12. The instrument was validated in a study by Pfingsten et $\mathrm{al}^{30}$ with 542 patients with different diagnoses.

Depressive symptoms were assessed by the German short version $^{31}$ of the Center for Epidemiological Studies Depression Scale, ${ }^{32}$ which is a 15 -item self-report scale from 0 (rarely) to 3 (most of the time), designed to measure depressive symptoms during the past 7 days. Validity and reliability (Cronbach's alpha $=0.91$ ) were good..$^{31}$

Catastrophizing cognitions concerning pain were measured with the German version of the Pain Catastrophizing Scale $^{33}$ (subscale "helplessness"). The subscale "helplessness" describes the feeling of the inability to cope with the pain. It consists of six items answered on a five-point scale ranging from 0 (not at all) to 4 (all the time). This subscale showed 
good internal consistency (Cronbach's alpha $=0.89$ ) as well as convergent validity. ${ }^{33}$

Pain acceptance was measured by ten items from the German version of the Chronic Pain Acceptance Questionnaire (items 1, 2, 6, 9, 11, 12, 13, 14, 15, 18). ${ }^{34}$ These items showed the highest correlation with the total score of the questionnaire. ${ }^{34}$ Items were answered on a seven-point scale ranging from 0 (never) to 6 (always), with an internal consistency of Cronbach's alpha $=0.73$.

\section{Statistical analysis}

One-way analyses of variance (ANOVAs), post-hoc Tukey's tests, and chi-square tests were conducted to explore differences between the four diagnostic groups regarding demographic and clinical variables.

To distinguish subgroups of patients with different levels of self-reported neuropathic sensory symptoms across the four diagnostic groups (clustering 1), a hierarchical cluster analysis including the seven sensory symptoms taken from the PDQ was performed. The commonly recommended hierarchical WARD-approach with a squared Euclidian distance measure was used. ${ }^{35}$ Agglomeration coefficients were investigated to establish the optimal cluster solution. The point at which the percentage of change was largest between steps determines the most appropriate cluster solution. ${ }^{36}$ A cutoff point for essential clusters was set at about $10 \%$ of evaluated cases. Multinominal regression analysis was conducted to examine associations between the levels of self-reported neuropathic sensory symptoms (clusters as predictors) with diagnostic group (criterion). Furthermore, ANOVAs were performed to investigate differences between these clusters regarding pain-related and psychological variables (separately for each diagnostic group as well as for total sample of).

For the purpose of identifying relevant subgroups of patients with different patterns of neuropathic sensory symptoms, a further hierarchical cluster analysis (clustering 2) was conducted on the basis of adjusted scores using the individual mean of the seven sensory symptoms (ie, the rating of each item by the patient was subtracted from the individual mean of all seven items rated by the same patient), thus eliminating the response bias of patients. This cluster analysis was followed by multiple discriminant analysis including the seven sensory symptoms as independent variables (criterion: clusters), to ensure the stability of the cluster solution. ${ }^{35}$ ANOVAs and chi-squared tests were performed to explore differences between the identified clusters regarding various pain and psychological variables as well as regarding their frequency in each diagnostic group. All analyses were conducted using SPSS (IBM Corporation, Armonk, NY, USA) software, version 19. The significance level was set at $P<0.05$.

\section{Results \\ Study sample}

Of the 344 patients, a total of 306 with TNP, RAD, FM, or nBP fulfilled the inclusion criteria. A total of 38 patients had to be excluded from the study: eleven patients because they had a pain history of less than 6 months, and 16 patients in whom tumor or other medical or psychiatric illness interfering with the pain assessment was diagnosed (eg, Alzheimer's disease or schizophrenia), six patients because of their inability to comprehend the German language, and five patients who refused to participate. The mean age of the patients was 59.2 years (standard deviation $[\mathrm{SD}]=13.2$ ). ANOVAs and post hoc tests showed significant differences between TNP and FM patients regarding age $(P=0.02)$, the latter being younger on average. The majority of patients were female $(65 \%)$. Separate chi-squared tests demonstrated a lower percentage of women with TNP compared with nBP $(P=0.007)$ and FM $(P=0.000)$ (Table 1). Also, results of the ANOVA and post hoc tests revealed significant differences between FM versus $\mathrm{nBP}$ and FM versus TNP regarding pain chronicity $(P=0.000)$ and between FM versus RAD and FM versus TNP regarding depressive symptoms $(P=0.006)$ (Table 1). The neuropathic characteristics (assessed by the PDQ total score) of nBP were significantly lower than in all other diagnostic groups $(P=0.02)$ (Table 1$)$. No group differences were found regarding pain history $(P=0.12)$, pain intensity $(P=0.69)$, pain acceptance $(P=0.07)$, or catastrophizing $(P=0.09)$ (Table 1).

\section{Cluster analysis I: Subgroups of patients with different levels of self-reported neuropathic sensory symptoms}

Based on the agglomeration coefficients for hierarchical cluster analysis, three distinct clusters emerged, which were characterized by a low $\left(\mathrm{M}_{\text {Low }}=1.38, \mathrm{SD}=0.69\right)$, moderate $\left(\mathrm{M}_{\text {Moderate }}=2.51, \mathrm{SD}=0.50\right)$, or high $\left(\mathrm{M}_{\mathrm{High}}=3.36, \mathrm{SD}=0.65\right)$ level of intensity of self-reported neuropathic sensory symptoms, which differed significantly from one another $(F(2,295)=267.72, P=0.000)$.

\section{Multinominal logistic regression analysis}

Multinominal logistic regression (criterion: diagnostic groups) with TNP as a reference group revealed that the 
Table I Demographic and clinical characteristics of patients (results of one-way ANOVA, post hoc Tukey's tests, and chi-squared test)

\begin{tabular}{|c|c|c|c|c|c|c|}
\hline Characteristic & $\begin{array}{l}\text { TNP } \\
\mathrm{n}=78\end{array}$ & $\begin{array}{l}\text { RAD } \\
n=68\end{array}$ & $\begin{array}{l}F M \\
n=6 I\end{array}$ & $\begin{array}{l}n B P \\
n=99\end{array}$ & $\begin{array}{l}F(d f) \text { or } \\
\chi^{2}(d f)\end{array}$ & $\begin{array}{l}\text { Tukey's test } \\
(P<0.05)\end{array}$ \\
\hline Age $(M \pm S D)$ & $61.05 \pm 12.8$ & $59.5 \pm 13.6$ & $54.78 \pm 10.1$ & $60.3 \pm 14.3$ & $F(3,302)=3.08^{*}$ & $\mathrm{FM}<\mathrm{TNP}$ \\
\hline \multicolumn{7}{|l|}{ Sex, n (\%) } \\
\hline Female & $47.4 \%$ & $64.8 \%$ & $82.5 \%$ & $67.7 \%$ & $\chi^{2}(3)=18.40^{* * *}$ & - \\
\hline Pain history (years) & $7.6 \pm 5.1$ & $7.3 \pm 5.6$ & $9.5 \pm 6.5$ & $8.9 \pm 8$ & $F(3,305)=1.75^{\dagger}$ & - \\
\hline NRS $(M \pm S D)$ & $6.63 \pm 1.9$ & $6.83 \pm 1.7$ & $6.91 \pm 1.6$ & $6.63 \pm 2.0$ & $F(3,300)=0.47^{\dagger}$ & - \\
\hline MPSS (M $\pm S D)$ & $8.72 \pm 1.1$ & $8.92 \pm 1.3$ & $9.28 \pm 1.1$ & $8.45 \pm 1.1$ & $F(3,288)=6.11 * * *$ & $\mathrm{nBP}, \mathrm{TNP}<\mathrm{FM}$ \\
\hline PDQ $(M \pm S D)$ & $19.01 \pm 7.2$ & $20.32 \pm 6.5$ & $19.50 \pm 6.9$ & $13.27 \pm 6.6$ & $F(3,299)=19.42^{* * *}$ & $\mathrm{nBP}<\mathrm{RAD}, \mathrm{TNP}, \mathrm{FM}$ \\
\hline $\mathrm{PCS}-\mathrm{H}(\mathrm{M} \pm \mathrm{SD})$ & $12.06 \pm 5.2$ & $10.93 \pm 5.7$ & $13.63 \pm 4.9$ & $12.33 \pm 5.2$ & $F(3,231)=1.84^{\dagger}$ & - \\
\hline CPAQ $(M \pm S D)$ & $32.93 \pm 10.1$ & $32.06 \pm 9.9$ & $27.4 I \pm 10.8$ & $29.64 \pm 9.6$ & $F(3,227)=2.46^{\dagger}$ & - \\
\hline ADS-K $(M \pm S D)$ & $15.63 \pm 8.9$ & $14.52 \pm 9.6$ & $21.93 \pm 10.1$ & $17.34 \pm 8.8$ & $F(3,230)=4.64 * *$ & RAD, TNP $<$ FM \\
\hline
\end{tabular}

Notes: $* * * P<0.001 ; * * P<0.01 ; * P<0.05 ;{ }^{\dagger}$ Not significant.

Abbreviations: ADS-K, Allgemeine Depressions Skala-Kurz version; ANOVA, analysis of variance; CPAQ, Chronic Pain Acceptance Questionnaire; df, degrees of freedom; FM, fibromyalgia; M, mean; MPSS, Mainz Pain Staging System; nBP, nociceptive back pain; NRS, Numeric Rating Scale; PCS-H, Pain Catastrophizing Scale-Helplessness; PDQ, Pain DETECT Questionnaire; RAD, radiculopathy; SD, standard deviation; TNP, typical neuropathic pain.

levels of self-reported neuropathic sensory symptoms only contributed to distinguishing TNP from $\mathrm{nBP}$ and not from RAD and FM. The model explained about $19 \%$ of the total variance (Table 2).

\section{Association of self-reported neuropathic sensory symptoms with pain and psychological variables in the four diagnostic groups}

Results of the ANOVA showed no significant differences between the three levels of neuropathic sensory symptoms regarding pain intensity, pain chronicity, pain catastrophizing, pain acceptance, and depressive symptoms (all $P>0.05$ ) in TNP, RAD, and FM (see Table 3 for more detail).

However, in nBP patients, significant differences were found regarding all variables, except pain chronicity (see Table 3 for further details). Accordingly, post hoc tests demonstrated significant differences between low versus high levels of neuropathic sensory symptoms for pain intensity and pain catastrophizing, between low versus moderate and high levels of neuropathic sensory symptoms for pain acceptance, and between low and moderate versus high levels of neuropathic sensory symptoms for depressive symptoms.
ANOVAs comparing all pain patients in the three levels of neuropathic sensory symptoms demonstrated significant differences regarding all variables, except with regard to pain catastrophizing (for details see Table 3). Post hoc tests also revealed significant differences between low and moderate versus high levels of neuropathic sensory symptoms for pain intensity, pain chronicity, and depressive symptoms and between low versus high levels of neuropathic sensory symptoms for pain acceptance.

\section{Cluster analysis 2: Subgroups of patients with distinct patterns of neuropathic sensory symptoms}

The cluster analysis based on adjusted scores of the seven sensory symptoms led to a four-cluster solution with distinct patterns of symptoms (Figure 1). Each of the four symptom patterns was present in every diagnostic group, differing only by relative frequency. Cluster 1 was characterized by a high intensity of prickling sensations $\left(\mathrm{M}_{\mathrm{adj}}=0.98\right)$, pain attacks $\left(\mathrm{M}_{\text {adj }}=0.98\right)$, and numbness $\left(\mathrm{M}_{\text {adj }}=0.62\right)$ compared with the other sensory symptoms. This pattern of symptoms occurred nearly two times more frequently in TNP $(26 \%)$ than in $\mathrm{FM}(15 \%)$, in $\mathrm{nBP}(13.3 \%)$, and in $\operatorname{RAD}\left(12.9 \% ; \chi^{2}=6.33\right.$,

Table 2 Multinominal logistic regression analysis: odds ratio, 95\% confidence intervals, significance, and pseudo $R^{2}$

\begin{tabular}{|c|c|c|c|c|}
\hline \multirow{2}{*}{$\begin{array}{l}\text { Level of self-reported } \\
\text { neuropathic sensory symptoms }\end{array}$} & \multicolumn{4}{|c|}{ Diagnostic group (Ref = typical neuropathic pain) } \\
\hline & RAD & FM & nBP & Pseudo $R^{2}$ \\
\hline Moderate level $(M=2.5 \mathrm{I})^{\mathrm{a}}$ & $1.06(0.37-3.05)^{\dagger}$ & $0.90(0.30-2.70)^{\dagger}$ & $0.24(0.10-0.55)^{* *}$ & \\
\hline High level $(M=3.36)^{a}$ & $0.86(0.28-2.64)^{\dagger}$ & $1.18(0.38-3.68)^{\dagger}$ & $0.05(0.01-0.16)^{* * * *}$ & $0.19 * * *$ \\
\hline Ref: Low level $(M=1.38)^{\mathrm{a}}$ & & & & \\
\hline
\end{tabular}

Notes: aMean score of the self-reported neuropathic sensory symptoms (sum of the seven sensory symptoms/7); ${ }^{* * * P}<0.00 \mathrm{I} ;{ }^{* * P}<0.01 ;{ }^{\dagger} \mathrm{Not}$ significant. Abbreviations: FM, fibromyalgia; M, mean; nBP, nociceptive back pain; RAD, radiculopathy; Ref, reference. 
Table 3 Means and SDs of various pain and psychological variables in patients with different levels of self-reported neuropathic sensory symptoms across the four diagnostic groups (clustering I)

\begin{tabular}{|c|c|c|c|c|c|c|}
\hline & & $\begin{array}{l}\text { "Cluster I" Low } \\
\text { level of neuropathic } \\
\text { sensory symptoms } \\
(\mathrm{M}=\mathrm{I.38})^{\mathrm{a}}\end{array}$ & $\begin{array}{l}\text { “Cluster 2" Moderate } \\
\text { level of neuropathic } \\
\text { sensory symptoms } \\
(\mathrm{M}=2.5 \mathrm{I})^{\mathrm{a}}\end{array}$ & 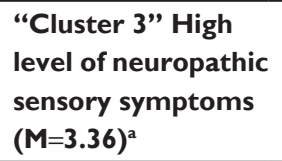 & $F(d f)$ & $\begin{array}{l}\text { Post hoc } \\
\text { "LSD" }\end{array}$ \\
\hline \multirow[t]{5}{*}{ TNP } & NRS $(M \pm S D)$ & $5.87 \pm 2.85$ & $6.4 I \pm I .75$ & $6.96 \pm 1.92$ & $1.15(2,70)^{\dagger}$ & - \\
\hline & MPSS $(M \pm S D)$ & $8.25 \pm 1.48$ & $8.73 \pm 1.05$ & $8.77 \pm 1.18$ & $0.68(2,70)^{\dagger}$ & - \\
\hline & $\mathrm{PCS}-\mathrm{H}(\mathrm{M} \pm \mathrm{SD})$ & $11.50 \pm 2.00$ & $12.86 \pm 5.70$ & $10.46 \pm 5.70$ & $0.88(2,40)^{\dagger}$ & - \\
\hline & CPAQ $(M \pm S D)$ & $33.80 \pm 10.29$ & $33.95 \pm 10.21$ & $32.64 \pm 11.18$ & $0.07(2,37)^{\dagger}$ & - \\
\hline & ADS-K $(M \pm S D)$ & $|5.00 \pm| .4 \mid$ & $16.72 \pm 9.72$ & $|5.2| \pm|0.4|$ & $0.16(2,40)^{\dagger}$ & - \\
\hline \multirow[t]{5}{*}{ RAD } & NRS $(M \pm S D)$ & $6.62 \pm 1.59$ & $6.68 \pm 1.77$ & $7.20 \pm 1.47$ & $0.69(2,63)^{\dagger}$ & - \\
\hline & MPSS $(M \pm S D)$ & $8.42 \pm 0.97$ & $8.89 \pm 1.44$ & $9.26 \pm 1.32$ & $1.03(2,61)^{\dagger}$ & - \\
\hline & $\mathrm{PCS}-\mathrm{H}(\mathrm{M} \pm \mathrm{SD})$ & $9.33 \pm 3.82$ & $|0.80 \pm 5.7|$ & $|2.23 \pm 6.2|$ & $0.66(2,55)^{\dagger}$ & - \\
\hline & $\mathrm{CPAQ}(\mathrm{M} \pm \mathrm{SD})$ & $37.66 \pm 12.45$ & $33.17 \pm 9.30$ & $27.58 \pm 10.11$ & $2.93(2,55)^{\dagger}$ & - \\
\hline & ADS-K $(M \pm S D)$ & $14.20 \pm 4.65$ & $12.82 \pm 9.33$ & $18.94 \pm 10.59$ & $2.39(2,54)^{\dagger}$ & - \\
\hline \multirow[t]{5}{*}{ FM } & NRS $(M \pm S D)$ & $6.28 \pm 1.60$ & $6.53 \pm 1.66$ & $7.50 \pm I .44$ & $3.03(2,55)^{\dagger}$ & - \\
\hline & MPSS $(M \pm S D)$ & $9.28 \pm 0.75$ & $8.92 \pm 1.23$ & $9.70 \pm 1.08$ & $3.04(2,55)^{\dagger}$ & - \\
\hline & $\mathrm{PCS}-\mathrm{H}(\mathrm{M} \pm \mathrm{SD})$ & $10.80 \pm 2.16$ & $13.40 \pm 5.47$ & $|6.37 \pm 4.7|$ & $2.11(2,25)^{\dagger}$ & - \\
\hline & $\mathrm{CPAQ}(\mathrm{M} \pm \mathrm{SD})$ & $31.40 \pm 11.80$ & $27.60 \pm 10.97$ & $26.14 \pm 11.52$ & $0.33(2,24)^{\dagger}$ & - \\
\hline & ADS-K $(M \pm S D)$ & $17.60 \pm 10.26$ & $20.53 \pm 9.54$ & $26.12 \pm 10.93$ & $1.28(2,25)^{\dagger}$ & - \\
\hline \multirow[t]{5}{*}{$n B P$} & NRS $(M \pm S D)$ & $6.06 \pm 1.82$ & $6.89 \pm 2.12$ & $8.00 \pm 2.23$ & $3.76(2,95)^{*}$ & $1<3$ \\
\hline & MPSS $(M \pm S D)$ & $8.33 \pm 1.16$ & $8.42 \pm 1.05$ & $9.14 \pm 1.06$ & $1.60(2,86)^{\dagger}$ & - \\
\hline & $\mathrm{PCS}-\mathrm{H}(\mathrm{M} \pm \mathrm{SD})$ & $11.04 \pm 5.99$ & $12.93 \pm 4.28$ & $16.00 \pm 4.20$ & $3.50(2,95)^{*}$ & $1<3$ \\
\hline & $\mathrm{CPAQ}(\mathrm{M} \pm \mathrm{SD})$ & $32.75 \pm 11.47$ & $27.91 \pm 6.85$ & $23.14 \pm 7.58$ & $5.00(2,95)^{* *}$ & $(2,3)<1$ \\
\hline & ADS-K $(M \pm S D)$ & $15.29 \pm 8.53$ & $|7.6| \pm 7.79$ & $25.85 \pm 10.63$ & $4.97(2,95)^{* *}$ & $(I, 2)<3$ \\
\hline \multirow[t]{5}{*}{ Total } & NRS $(M \pm S D)$ & $6.19 \pm 1.93$ & $6.65 \pm 1.86$ & $7.25 \pm 1.68$ & $6.09(2,293)^{* *}$ & $(I, 2)<3$ \\
\hline & MPSS $(M \pm S D)$ & $8.44 \pm 1.15$ & $8.72 \pm 1.21$ & $9.22 \pm 1.22$ & $7.76(2,28 I)^{* * * *}$ & $(I, 2)<3$ \\
\hline & $\mathrm{PCS}-\mathrm{H}(\mathrm{M} \pm \mathrm{SD})$ & $10.96 \pm 5.18$ & $12.35 \pm 5.19$ & $13.02 \pm 5.93$ & $2.22(2,224)^{\dagger}$ & - \\
\hline & $\mathrm{CPAQ}(\mathrm{M} \pm \mathrm{SD})$ & $33.26 \pm 10.91$ & $30.50 \pm 9.15$ & $28.06 \pm 10.60$ & $3.57(2,220)^{*}$ & $3<1$ \\
\hline & ADS-K $(M \pm S D)$ & $15.38 \pm 7.77$ & $16.41 \pm 9.11$ & $20.17 \pm 11.27$ & $3.82(2,223)^{*}$ & $(I, 2)<3$ \\
\hline
\end{tabular}

Notes: aMean score of the self-reported neuropathic sensory symptoms (sum of the 7 sensory symptoms/7); $* * * P<0.001 ; * * P<0.01 ; * P<0.05 ;{ }^{\dagger}$ Not significant.

Abbreviations: ADS-K, Allgemeine Depressions Skala-Kurz version; CPAQ, Chronic Pain Acceptance Questionnaire; df, degrees of freedom; FM, fibromyalgia; LSD, Fisher's least significant difference; M, mean; MPSS, Mainz Pain Staging System; nBP, nociceptive back pain; NRS, Numeric Rating Scale; PCS-H, Pain Catastrophizing Scale-Helplessness; RAD, radiculopathy; SD, standard deviation; TNP, typical neuropathic pain.

degrees of freedom $[\mathrm{df}]=3, P=0.09$ ) (Figure 1). Patients who had been classified into cluster 2 reported particularly high levels of pain attacks $\left(\mathrm{M}_{\text {adj }}=1.56\right)$ and pressure hyperalgesia $\left(\mathrm{M}_{\mathrm{adj}}=0.97\right)$. All other symptoms were close to the mean, except burning pain, which was less severe. In patients with nBP (44.9\%), FM (38.3\%), and RAD (28.6\%), this symptom pattern was more frequent than in TNP patients $\left(14.3 \% ; \chi^{2}=23.87, \mathrm{df}=3, P=0.000\right)$ (Figure 1). Cluster 3 was characterized by an overall "flat profile" (ie, no item deviated much from the individual mean) (Figure 1). This pattern of symptoms evenly distributed over all four diagnostic groups $\left(\chi^{2}=6.06, \mathrm{df}=3, P=0.10\right)$ (Figure 1). The dominant symptom of cluster 4 was a severe burning pain $\left(\mathrm{M}_{\text {adj }}=2.05\right)$. Pain attacks $\left(\mathrm{M}_{\mathrm{adj}}=0.98\right)$ and thermal pain $\left(\mathrm{M}_{\mathrm{adj}}=0.67\right)$ were also above the individual mean. All other symptoms were below average. This symptom pattern prevailed in TNP (19.5\%), in RAD (15.7\%), and in FM (13.3\%) (Figure 1).
A discriminant analysis with the seven neuropathic sensory symptoms as independent variables (criterion: the four patterns of neuropathic sensory symptoms) led to three significant discriminating functions $(P=0.000$ for each function) with Wilks' lambda of $0.16,0.36$, and 0.63 and eigenvalues of 1.24, 0.75, and 0.57. Eighty-eight percent of the patients were classified correctly. Burning pain $(\beta=0.99)$, thermal hyperalgesia $(\beta=0.91)$, and pain attacks $(\beta=0.84)$ were the best predictors for the clusters (symptom patterns). But also, prickling sensations $(\beta=0.56)$, pressure hyperalgesia $(\beta=0.55)$, numbness $(\beta=0.48)$, and allodynia $(\beta=0.40)$ contributed significantly to the discrimination of the four clusters.

The results of the ANOVAs showed no significant differences regarding pain intensity $(P=0.95)$, pain chronicity ( $P=0.11)$, pain catastrophizing $(P=0.87)$, pain acceptance ( $P=0.46)$, and depressive symptoms $(P=0.78)$ when comparing the four clusters based on adjusted scores. 


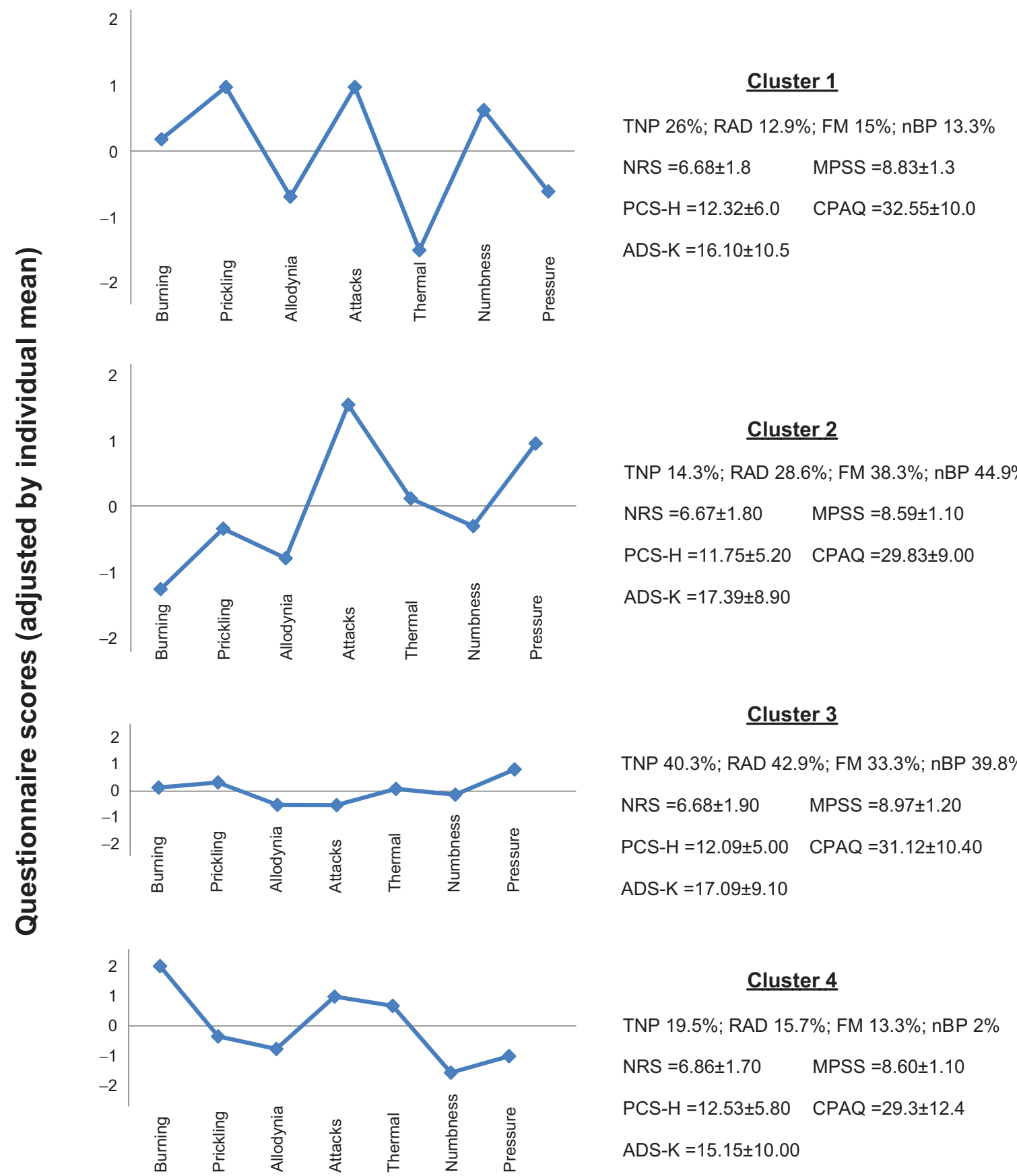

Figure I Subgroups of chronic pain patients with distinct patterns of neuropathic sensory symptoms using the Pain DETECT Questionnaire (clustering 2). Note: Vertical axis: adjusted scores of neuropathic sensory symptoms; horizontal axis marked as 0 : average of seven adjusted scores of neuropathic sensory symptoms. Abbreviations: ADS-K, Allgemeine Depressions Skala-Kurz version; CPAQ, Chronic Pain Acceptance Questionnaire; FM, fibromyalgia; MPSS, Mainz Pain Staging System; nBP, nociceptive back pain; NRS, Numeric Rating Scale; PCS-H, Pain Catastrophizing Scale-Helplessness; RAD, radiculopathy; TNP, typical neuropathic pain.

\section{Discussion}

The present study demonstrates that neither the intensity of the neuropathic sensory symptoms nor any patterns of these symptoms exclusively lead to a high degree of pain intensity, pain chronicity, and negative affectivity in a clinical sample of patients with underlying pathology of neuropathic sensory symptoms (ie, TNP, RAD, and FM). Our findings also highlight the fact that high ratings of self-reported neuropathic sensory symptoms are not necessarily associated with major neuropathic characteristics of pain but are also related to a negative response tendency in patients without any identifiable underlying pathology of neuropathic sensory symptoms (ie, nBP). These results demonstrate the importance of taking advantage of response bias-adjusted scores 
when investigating self-reported neuropathic symptoms by patients.

The assumption that neuropathic sensory symptoms would differentiate TNP from nBP was confirmed by the results of regression analysis (Table 2). Patients medically diagnosed with $\mathrm{nBP}$ also revealed the lowest neuropathic score assessed by the PDQ, differing significantly from all other diagnostic groups (TNP, RAD, FM), which are supposed to present pain with underlying pathology of neuropathic sensory symptoms (Table 1). Neuropathic sensory symptoms, however, did not distinguish TNP from FM. Although in the majority of FM patients no nerve lesion can be detected, ${ }^{37}$ numerous studies have demonstrated similarities between neuropathic pain patients and FM patients with regard to the experience of sensory symptoms. ${ }^{3}$ Vierck $^{37}$ suggested that changes in the milieu of muscles and other deep somatic structures in FM might lead to a similar state of hyperactivity in nociceptive neurons as is observed after nerve damage. Moreover, Staud et $\mathrm{al}^{38}$ using functional MRI, provided strong support for neuroplastic changes in the central nervous system in FM. However, it must be stressed that the classification of FM as a variant of neuropathic pain is a subject of controversy among researchers. ${ }^{1,17}$

As hypothesized, the three levels of self-reported neuropathic sensory symptoms in chronic pain patients with underlying pathology of neuropathic sensory symptoms did not differ in pain intensity and chronicity, depression, pain acceptance, and catastrophizing. Thus, neuropathic sensory symptoms do not contribute per se to the characteristics of pain-related features and cognitive-emotional processes. This interpretation finds support in some clinical studies $^{8-10}$ as well as our own findings (Table 1), indicating no significant differences between medically diagnosed neuropathic and non-neuropathic pain regarding pain intensity, pain chronicity, and psychological factors. Other possible explanations for this result may be that negative emotions and maladaptive appraisals do not play an important role in the report of sensory descriptors of pain such as neuropathic sensory symptoms. In accordance with this interpretation, Sullivan et $\mathrm{al}^{39}$ found that pain catastrophizing in neuropathic pain patients was not associated with the sensory subscale of the McGill Pain Questionnaire but only with the affective aspects. They suggested that the mechanism of pain catastrophizing is specifically related to the affective dimension of pain. Functional imaging studies with FM have also demonstrated that neither a high level of pain catastrophizing ${ }^{40}$ nor the presence of a clinically diagnosed major depression ${ }^{41}$ are associated with the sensory aspects of pain processing.
In contrast to this finding, in patients with nBP, an association between high levels of neuropathic sensory symptoms with high levels of pain intensity, depression, pain catastrophizing, and with less acceptance of pain was observed, suggesting a general negative response tendency among patients with nBP who scored high on neuropathic sensory symptoms without any identifiable underlying neuropathology. Ambiguity about the origin and significance of neuropathic sensory symptoms among patients with nBP may explain the biased response tendency regarding these symptoms, particularly among those nBP patients with negative affect and self-appraisals. According to Social Comparison Theory $^{42}$ and Temporal Comparison Theory, ${ }^{43,44}$ individuals need comparison standards to evaluate their opinions, skills, and social status, as well as their physical state. Petersen et $\mathrm{al}^{45}$ proposed the comparison standards as a predictor of symptom presentations and contended that in evaluating a bodily state, individuals have to use reference standards, such as the personal experience of symptoms in the past, or beliefs about the perception of sensations by relevant others, such as patients or healthy individuals. It can be therefore argued that those nBP patients with negative affect and selfappraisals are most likely influenced by a biased response tendency regarding neuropathic sensory symptoms. The finding that the three levels of neuropathic sensory symptoms in nBP did not differentiate pain chronicity which was not obtained by self-report may provide additional evidence for the argument above.

Altogether, our findings suggest that a high level of neuropathic sensory symptoms in patients with underlying pathology of neuropathic sensory symptoms does not automatically result in a high intensity of pain-related characteristics and psychological dysfunctional features.

With the second clustering approach, response biasadjusted scores were created. This enabled us to characterize different subgroups of patients with common patterns of neuropathic sensory symptoms. Consistent with earlier studies, ${ }^{9}$ none of the symptom patterns was exclusively seen in the groups with diagnosed neuropathic or nociceptive pain. Nevertheless, the distributions of symptom patterns differed between different diagnostic groups. For example, symptom pattern 4 occurred only in $2 \%$ of the patients with $\mathrm{nBP}$, whereas nearly half of the nBP patients demonstrated the symptom pattern characterized by a high level of pain attacks and pressure hyperalgesia (cluster 2 ). In any case, we found that neither the symptom patterns frequently occurring in neuropathic pain nor symptom patterns frequently occurring in nBP were associated with a higher level of painrelated and psychological factors. This finding gives reason to 
further question a genuine association of neuropathic sensory symptoms with high levels of pain and psychological factors in patients with underlying pathology of neuropathic sensory symptoms. At the same time, it highlights the adequacy of our strategy for analysis.

Intriguingly, about $40 \%$ of the chronic pain patients showed a "flat profile" of neuropathic sensory symptoms. The "flat profile" indicates that the patient responded in a similar way to all items of the PDQ. This pattern was demonstrated by a similar percentage of subgroups of patients from the four diagnostic groups, a finding that has been reported before. ${ }^{46}$ Possibly, a psychological phenomenon (ie, the tendency to adhere to a similar rating style regardless of item content) is responsible for this observation. For a more accurate interpretation, we compared patients demonstrating a "flat profile" with different levels of neuropathic sensory symptoms (in clustering 1). Interestingly, among nBP patients who scored "high" (in clustering 1) as well as "flat" on neuropathic sensory symptoms, we found a higher level of depressive symptoms $(P=0.004)$ and pain catastrophizing $(P=0.04)$ as well as less acceptance of pain $(P=0.04)$. Consistent with this finding, Cohen et $\mathrm{al}^{47}$ proposed that negative emotions may not only result in a negative response bias as discussed before but also lead to an undifferentiated interpretation of symptoms. Such differences were not found regarding TNP, RAD, and FM with a "flat" and also "high" pattern of neuropathic sensory symptoms. This result can be interpreted that a "flat and high" pattern of neuropathic sensory symptoms is not necessarily based on a response bias, particularly in the case of symptoms supported by underlying pathology.

Before considering the implications of our findings, several limitations of this study have to be acknowledged. The sample size was relatively small and thus the reliability of the findings has to be questioned until other studies can replicate them. An additional limitation relates to the applied instrument, since the PDQ has not been validated regarding FM. Furthermore, our sample of patients was recruited from a single clinic, and this selection might have affected the results.

In summary, our data seem to highlight the conclusion that neuropathic sensory symptoms alone are not sufficient to produce a high degree of pain and psychological dysfunctional features as it is not the case in patients with underlying pathology of neuropathic sensory symptoms. Moreover, the present study corroborates previous findings that response bias may more strongly relate to the report of symptoms without any identified pathology as well as symptoms which were not experienced by patients in the past than with symptoms that can be verified by clinical examination or medical tests. These findings should have implications for symptom-based studies of neuropathic pain to include a relevant sample of patients when investigating the association of these symptoms with other indicators of health status. The results also highlight the importance of using adjusted scores to eliminate the response bias when investigating selfreported symptoms by patients.

\section{Author contributions}

All of the authors contributed to the study design, statistical analysis and data interpretation; patient selection and examination of patients; drafting the article or revising it critically for important intellectual content.

\section{Acknowledgments}

This research was supported by the German Research Foundation and the Open Access Publication Funds of the Göttingen University. We would like to thank Dr Antonia Barke for her support in giving valuable advice with regard to the publication.

\section{Disclosure}

The authors report no conflicts of interest in this work.

\section{References}

1. Treede RD, Jensen TS, Campbell JN, et al. Neuropathic pain: redefinition and a grading system for clinical and research purposes. Neurology. 2008; 70:1630-1635.

2. Boureau F, Doubrhe JF, Luu M. Study of verbal description in neuropathic pain. Pain. 1990;42:145-152.

3. Koroschetz J, Rehm SE, Gockel U, et al. Fibromyalgia and neuropathic pain-differences and similarities. A comparison of 3057 patients with diabetic painful neuropathy and fibromyalgia. BMC Neurol. 2011;11: $55-62$.

4. Freynhagen R, Baron R, Gockel U, Tölle TR. pain DETECT: a new screening questionnaire to identify neuropathic components in patients with back pain. Curr Med Res Opin. 2006;22:1911-1920.

5. Torrance N, Smith BH, Bennett MI, Lee AJ. The epidemiology of chronic pain of predominantly neuropathic origin. Results from a general population survey. J Pain. 2006;7:281-289.

6. Bouhassira D, Lantéri-Minet M, Attal N, Laurent B, Touboul C. Prevalence of chronic pain with neuropathic characteristics in the general population. Pain. 2008;136:380-387.

7. Attal N, Lanteri-Minet M, Laurent B, Fermanian J, Bouhassira D. The specific disease burden of neuropathic pain: results of a French nationwide survey. Pain. 2011;152:2836-2843.

8. Bennett M. The LANSS Pain Scale: the Leeds assessment of neuropathic symptoms and signs. Pain. 2001;92:147-157.

9. Dworkin RH, Jensen MP, Gammaitoni AR, Olaleye DO, Galer BS. Symptom profiles differ in patients with neuropathic versus nonneuropathic pain. J Pain. 2007;8:118-126.

10. Daniel HC, Narewska J, Serpell M, Hoggart B, Johnson R, Rice AS. Comparison of psychological and physical function in neuropathic pain and nociceptive pain: Implications for cognitive behavioral pain management programs. Eur J Pain. 2008;12: 731-741. 
11. Freynhagen R, Baron R. The evaluation of neuropathic components in low back pain. Curr Pain Headache Rep. 2009;13:185-190.

12. Baron R, Binder A. Wie neuropathisch ist die Lumboischialgie? [Is sciatica neuropathic? The mixed pain concept]. Orthopäde. 2004;33:568-575. German.

13. Deyo RA, Weinstein JN. Low back pain. $N$ Engl J Med. 2001;344: 363-370.

14. Uceyler N, Zeller D, Kahn AK, et al. Small fibre pathology in patients with fibromyalgia syndrome. Brain. 2013;136:1857-1867.

15. Kosek E, Ekholm J, Hansson P. Sensory dysfunction in fibromyalgia patients with implications for pathogenic mechanisms. Pain. 1996;68: 375-383.

16. Bradley LA, McKendree-Smith NL, Alarcon GS, Cianfrini LR. Is fibromyalgia a neurologic disease? Curr Pain Headache Rep. 2002; 6(2):106-114.

17. Crofford LJ. The relationship of fibromyalgia to neuropathic pain syndromes. J Rheumatol Suppl. 2005;75:41-45.

18. Elliott MN, Haviland AM, Kanouse DE, Hambarsoomian K, Hays RD. Adjusting for subgroup differences in extreme response tendency in ratings of health care: impact on disparity estimates. Health Serv Res. 2009; $44: 542-561$.

19. Feldman P, Cohen S, Doyle WJ, Skoner DP, Gwaltney JM Jr. The impact of personality on the reporting of unfounded symptoms and illness. J Pers Soc Psychol. 1999;77:370-378.

20. Kolk AMM, Hanewald GJFP, Schagen S, Gijsbers van Wijk CMT. Predicting medically unexplained physical symptoms and health care utilization: a symptom-perception approach. J Psychosom Res. 2002; 52:35-44.

21. Cohen S, William JD, Ronald BT, Cuneyt MA, David PS. Emotional style and susceptibility to the common cold. Psychosom Med. 2003; 65:652-657.

22. Förster M, Mahn F, Gockel U, et al. Axial low back pain: one painful area-many perceptions and mechanisms. PLoS One. 2013;8: e68273.

23. Harden RN, Bruehl SP. Diagnosis of complex regional pain syndrome: signs, symptoms, and new empirically derived diagnostic criteria. Clin J Pain. 2006;22:415-419.

24. England JD, Gronseth GS, Franklin G, et al. Distal symmetric polyneuropathy: a definition for clinical research: report of the American Academy of Neurology, the American Association of Electrodiagnostic Medicine, and the American Academy of Physical Medicine and Rehabilitation. Neurology. 2005;64:199-207.

25. Manzoni GC, Torelli P, International Headache Society. International Headache Society classification: new proposals about chronic headache. Neurol Sci. 2003;24 Suppl 2:86-89.

26. Wolfe F, Smythe HA, Yunus MB, et al. The American College of Rheumatology 1990 criteria for the classification of fibromyalgia. Report of the Multicenter Criteria Committee. Arthritis Rheum. 1990; $33: 160-172$

27. International Statistical Classification of Disease and Related Health Problems 10. Revision, Geneva, Switzerland, World Health Organization, German Modification, 2012.

28. Dworkin RH, Turk DC, Farrar JT, et al. Core outcome measures for chronic pain clinical trials: IMMPACT recommendations. Pain. 2005; 113:9-19.

Neuropsychiatric Disease and Treatment

\section{Publish your work in this journal}

Neuropsychiatric Disease and Treatment is an international, peerreviewed journal of clinical therapeutics and pharmacology focusing on concise rapid reporting of clinical or pre-clinical studies on a range of neuropsychiatric and neurological disorders. This journal is indexed on PubMed Central, the 'PsycINFO' database and CAS,
29. Gerbershagen HU, Lindena G, Korb J, Kramer S. Health-related quality of life in patients with chronic pain. Schmerz. 2002;16:271-284.

30. Pfingsten M, Schöps P, Wille T, Terp L, Hildebrandt J. Classification of chronic pain. Quantification and grading with the Mainz Pain Staging System. Schmerz. 2000;14(1):10-17.

31. Hautzinger M, Bailer M. Allgemeine Depressionsskala [General Depression Scale]. 1. Auflage. Göttingen, Germany: Beltz Test GmbH; 1993. German.

32. Radloff LS. The CES-D scale: a self-report depression scale for research in the general population. Appl Psychol Meas. 1977;1:385-401.

33. Meyer K, Sprott H, Mannion AF. Cross-cultural adaptation, reliability, and validity of the German version of the pain catastrophizing scale. J Psychosom Res. 2008;64:469-478.

34. Nilges $P$, Köster B, Schmidt CO. Schmerzakzeptanz-Konzept und Überprüfung einer deutschen Fassung des Chronic Pain Acceptance Questionnaire [Pain acceptance - concept and validation of a German version of the Chronic Pain Acceptance Questionnaire]. Schmerz. 2007;21:57-67. German.

35. Punj G, Stewar DW. Cluster analysis in marketing research: review and suggestions for application. J Marketing Res. 1983;20:134-148.

36. Milligan GA, Cooper MC. An examination of procedures for determining the number of clusters in a data set. Psychometrika. 1967;50: 159-179.

37. Vierck CJ Jr. Mechanisms underlying development of spatially distributed chronic pain (fibromyalgia). Pain. 2006;124(3):242-263.

38. Staud R, Craggs JG, Perlstein WM, Robinson ME, Price DD. Brain activity associated with slow temporal summation of C-fiber evoked pain in fibromyalgia patients and healthy controls. Eur J Pain. 2008; 12:1078-1089.

39. Sullivan MJL, Lynch ME, Clark AJ. Dimensions of catastrophic thinking associated with pain experience and disability in patients with neuropathic pain conditions. Pain. 2005;113:310-315.

40. Gracely RH, Geisser ME, Giesecke T, et al. Pain catastrophizing and neural responses to pain among persons with fibromyalgia. Brain. 2004; 127:835-843.

41. Giesecke T, Williams DA, Harris RE, et al. Subgrouping of fibromyalgia patients on the basis of pressure-pain thresholds and psychological factors. Arthritis Rheum. 2003;48:2916-2922.

42. Festinger L. A theory of social comparison processes. Hum Relat. 1954;7: 117-140.

43. Albert S. Temporal comparison theory. Psychol Rev. 1977;84:485-503.

44. Zell E, Alicke MD. Self-evaluative effects of temporal and social comparison. J Exp Soc Psychol. 2009;45:223-227.

45. Petersen S, van den Berg RA, Janssens T, van den Berg O. Illness and symptom perception: a theoretical approach towards an integrative measurement model. Clin Psychol Rev. 2011;31:428-439.

46. Baron R, Tölle TR, Gockel U, Brosz M, Freynhagen R. A cross-sectional cohort survey in 2100 patients with painful diabetic neuropathy and postherpetic neuralgia: differences in demographic data and sensory symptoms. Pain. 2009;146:34-40.

47. Cohen S, Doyle WJ, Skoner DP, Fireman P, Gwaltney JM, Newsom JT. State and trait negative affect as predictors of objective and subjective symptoms of respiratory viral infections. J Pers Soc Psychol. 1995; 68:159-169.

\section{Dovepress}

and is the official journal of The International Neuropsychiatric Association (INA). The manuscript management system is completely online and includes a very quick and fair peer-review system, which is all easy to use. Visit http://www.dovepress.com/testimonials.php to read real quotes from published authors. 